\title{
What Is Your Diagnosis?
}

In collaboration with the American College of Veterinary Radiology

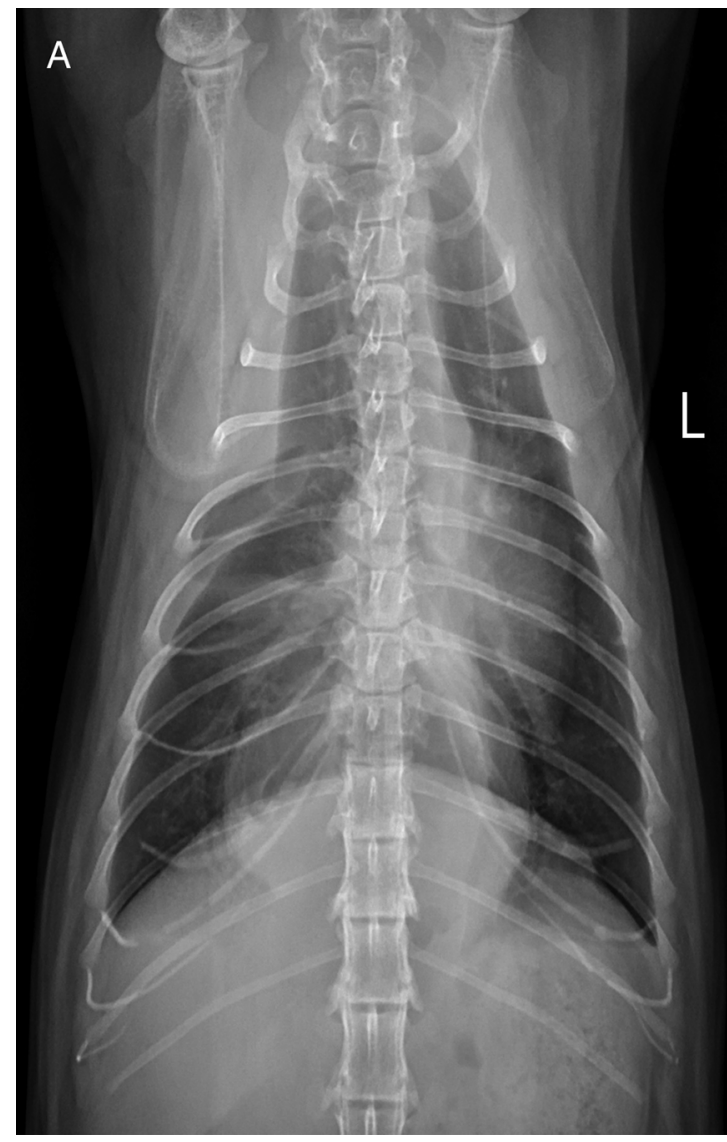

\section{History}

A 12-year-old 3.8-kg castrated male chinchilla Persian cat with a 2-day history of anorexia and tachypnea after having been in a catfight. Findings on physical examination and thoracic auscultation of the cat were unremarkable. Routine hematologic and serum

Yi-Chun Lin, DVM'; Hock Gan Heng, DVM, MVS, MS²; Wan-Ching Cheng, DVM, PhD'; Ping Wei, DVM'; Kuan-Sheng Chen, DVM, $\mathrm{PhD}^{1,3 *}$

IVeterinary Medical Teaching Hospital, College of Veterinary Medicine, National Chung Hsing University, Taichung, Taiwan

${ }^{2}$ VetCT, Orlando, FL

${ }^{3}$ Department of Veterinary Medicine, College of Veterinary Medicine, National Chung Hsing University, Taichung, Taiwan

*Corresponding author: Dr. Chen (kschen@dragon.nchu.edu.tw)

https://doi.org/I0.2460/javma.21.07.0333

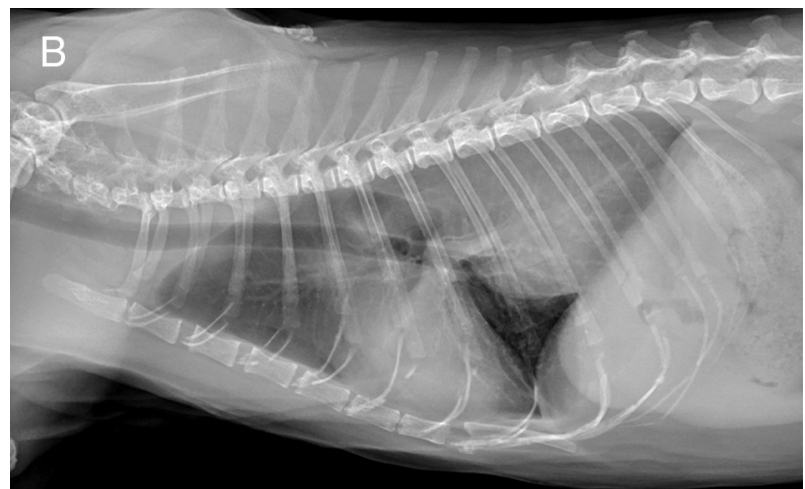

Figure I-Ventrodorsal (A) and right lateral (B) thorax radiographic images of 12 -year-old $3.8-\mathrm{kg}$ castrated male chinchilla Persian cat with 2 days history of anorexia and tachypnea after having been in a catfight.

biochemical analyses revealed no abnormalities except for mild anemia (Hct, 26.4\%; reference range, 30.3\% to 52.3\%). Thoracic radiography (Figure I) and abdominal radiography (not shown) were performed.

Formulate differential diagnoses, then continue reading.

\section{Diagnostic Imaging Findings and Interpretation}

On thoracic radiography, an oval, soft tissue mass was in the mid caudodorsal region of the thorax (Figure 2). The mass displaced the heart leftward. The dorsal border of the mass was adjacent to the ventral aspect of the thoracic vertebrae. The diaphragm could be traced, which suggested an inherent contrast between the composition of the mass (fat component) and other soft tissue, whereas the effacement of the caudal vena cava and descending aorta indicated a soft tissue component. There was moderate retraction of the right cranial lung lobe from the thoracic wall, with a moderate amount of soft tissue opaque structure between them, and widening of the interlobar fissure between the right middle and right caudal lung lobes was identified. Findings on abdominal radiography were unremarkable, and no displacement of abdominal organs was observed.

The origin of the mass in the mid caudodorsal region of the thorax could have been from the caudal aspect of the mediastinum, such as a lipoma with necrosis, hemorrhage, or both; liposarcoma; omental 

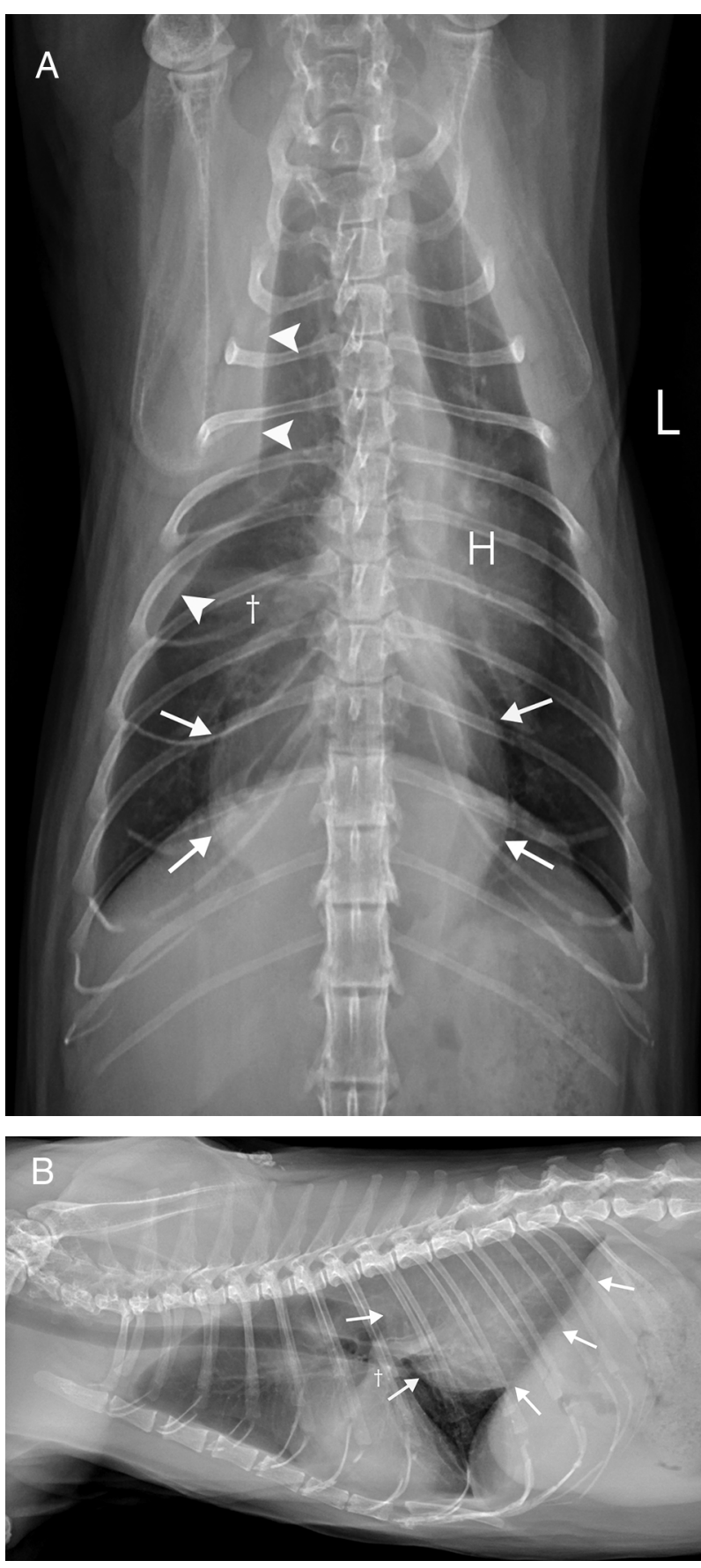

Figure 2-Same as in Figure I. A mass (arrows) with the composition of fat and soft tissue is in the caudodorsal aspect of the thorax on midline. The heart $(\mathrm{H})$ is displaced leftward, and the right cranial and right middle lung lobes are retracted from the thoracic wall with interposed soft tissue opacity, indicating a moderate amount of pleural fluid (arrowheads). There is soft tissue to fat opaque structure or trapped pleural fluid (daggers) between the right middle and right caudal lung lobes.

herniation with inflammation or hemorrhage; or, less likely, a cyst, abscess, paraesophageal empyema, or esophageal granuloma. Other causes considered less likely included primary neoplasia of the esophagus, accessory lung lobe, primary diaphragmatic neoplasia, or paraesophageal hernia. The presence of pleural fluid in the cranial aspect of the right hemithorax could have been from the accumulation of inflammatory effusion, neoplastic effusion, hemorrhage, or, less likely, chyle or inflammatory exudate. Thoracic and abdominal CT were recommended; however, the owner declined, and the cat was sent home with a prescription of piroxicam $(0.3 \mathrm{mg} / \mathrm{kg}, \mathrm{PO}, \mathrm{q} 24 \mathrm{~h})$.

The cat's condition did not improve, and 4 days later, pre- and postcontrast CT were performed. Between the left and right diaphragmatic crus was a 3 -cm-wide defect, through which omental fat (HU, -127 to -109$)$ herniated into the caudodorsal region of the thorax (Figure 3). The herniated omental fat appeared as a $6 \times 5.4 \times 4.3-\mathrm{cm}$ mass and contained soft tissue-attenuating striations and regions. Some of the striations and regions were noncontrast enhancing, indicative of steatitis or the presence of a small amount of fluid (fat stranding), whereas others were contrast enhancing, indicative of blood vessels from the splenic or left gastroepiploic vessel. The herniated omental fat compresses and displaces the caudal vena cava ventrally and the esophagus toward the left. The fundus of the stomach is mildly displaced cranially and leftward, adjacent to the left crus of the diaphragm and the abdominal wall. There is a thin soft tissue-attenuating hernia sac covering the herniated contents, and disruption or discontinuity of the right craniodorsal aspect of the hernia sac was identified. Herniated omental fat extended through the disrupted hernia sac, occupied approximately twothirds of the right pleural cavity, and caused compressive atelectasis in the right cranial, right middle, and right caudal lung lobes. The soft tissue opacity identified radiographically between the right middle and right caudal lung lobes was confirmed on CT to have been herniated omental fat (extended to the third intercostal space) and a small amount of sequestered pleural fluid cranial to it. The pleural fluid identified unilaterally on radiographic examination was identified bilaterally on CT.

\section{Treatment and Outcome}

Ultrasound-guided fine-needle aspirate samples of the herniated omental fat in the mid caudodorsal region of the thorax and pleural fluid from the right pleural cavity were obtained immediately after CT. Cytologic examination revealed that the intrathoracic mass was granulomatous steatitis, and fluid analysis revealed inflammatory effusion with nondegenerate neutrophils and macrophages. Based on these findings, the final diagnosis was confirmed to have been diaphragmatic herniation of the inflamed and granulomatous omental fat with secondary pleural exudate.

The owner declined surgical intervention. Anti-inflammatory (prednisolone; $1.5 \mathrm{mg} / \mathrm{kg}$, PO, q $24 \mathrm{~h}$ for 4 weeks and then q 3 days for 4 weeks) and antioxidative (vitamin E, $50 \mathrm{mg}$, PO, q $24 \mathrm{~h}$, for 4 weeks) treatments were prescribed, and the patient 

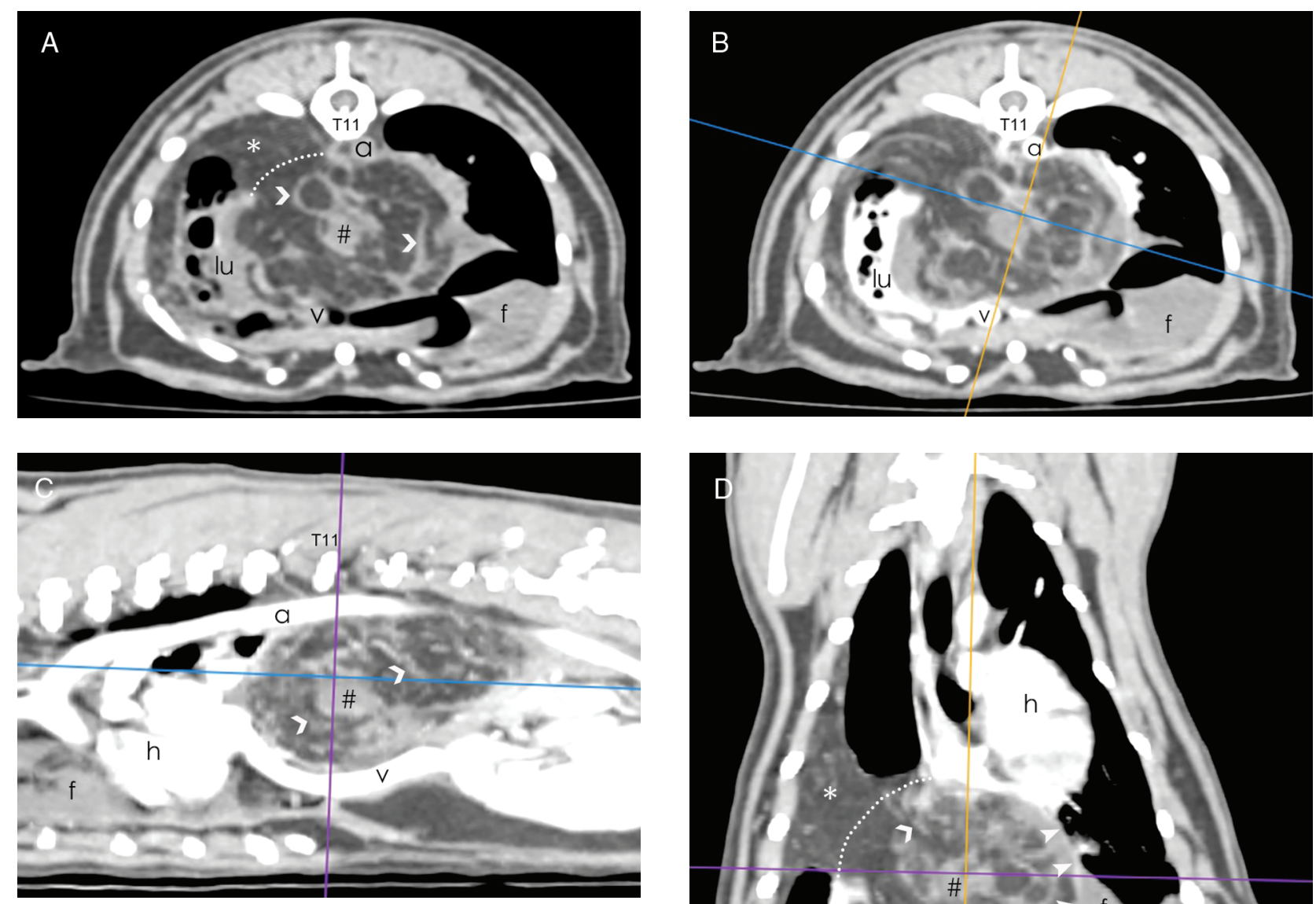

Figure 3-Precontrast transverse $(\mathrm{A})$ and postcontrast multiplanar reconstructed transverse (B), oblique sagittal (C), and oblique dorsal (D) plane caudal thoracic and cranial abdominal CT images of the cat described in Figure I, revealing a diaphragmatic defect (dotted straight line) through which a hernia sac (arrowheads) containing omental fat herniates into the caudodorsal aspect of the thorax, displaces the caudal vena cava (v) ventrally, and causes compressive atelectasis of lung lobes (lu). The hernia sac is disrupted along the right craniolateral aspect (dotted curved line), and herniated omental fat is in the right pleural space (asterisks). In addition to fat-attenuating tissue in the hernia sac, the sac contains non-contrast-enhancing soft tissue-attenuating striations

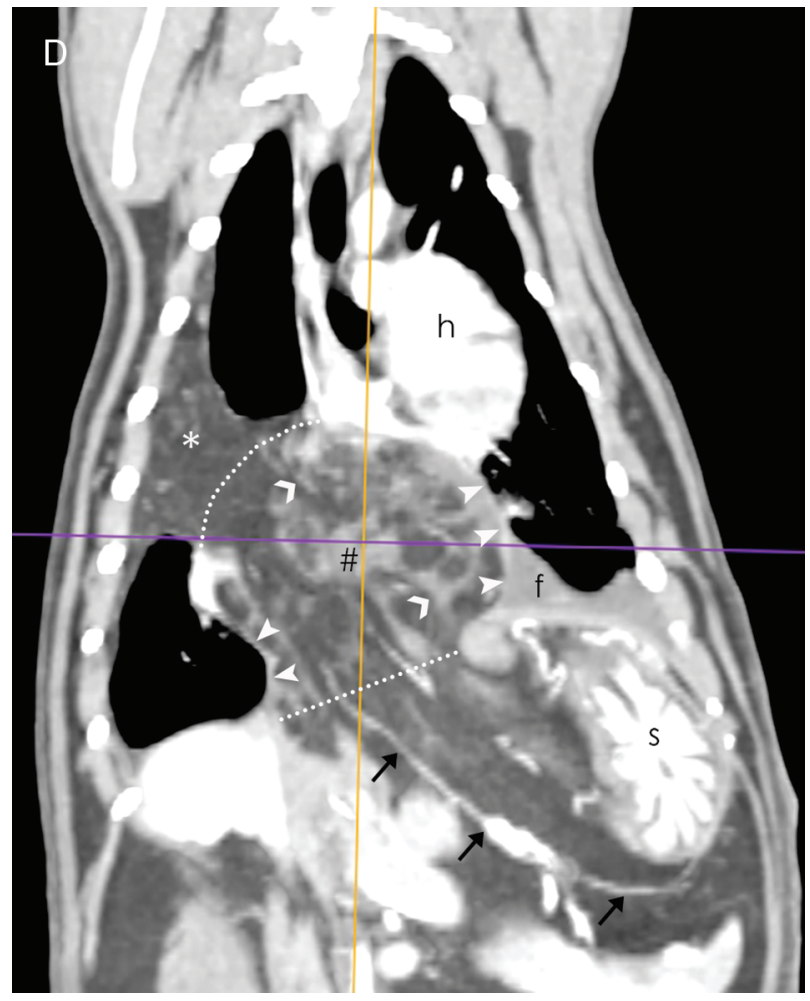
(chevrons) and a soft tissue component (pound signs), indicative of inflammation or fluid as well as contrast-enhancing components, indicative of blood vessels from the splenic or left gastroepiploic vessel. There is a moderate amount of pleural fluid (f), the stomach (s) is displaced craniolaterally, and contrast-enhanced omental vessels (eg, gastrosplenic vessels [arrows]) are visible. In all images, the cat's right side (A, B, and D) or head (C) is toward the left. The lines (purple, yellow, and blue) across images represent the planes of other images $(A$ and $B, C$, and $D$, respectively). $a=$ Aorta. $h=H$ Heart. TII $=I I$ th thoracic vertebra.

was discharged for home care and monitoring. No abnormal cardiovascular or respiratory signs were observed, and radiographic examination 1 year later showed reduced herniated omental fat and pleural fluid (Figure 4).

\section{Comments}

Omental fat herniation into the thorax is rare in cats and humans. To our knowledge, only 1 report describes omental herniation through the esophageal hiatus in a cat, ${ }^{1}$ and there are a few case reports of such herniation in humans. ${ }^{2,3}$ Risk factors of omental herniation through esophageal hiatus in people include increased age, obesity, and pregnancy, which might be associated with a reduction of elastic fibers in the phrenoesophageal membrane. ${ }^{3}$ The commonalities between the cat of the present report and the affected cat previously reported ${ }^{1}$ were older age and omental fat herniation into the mid caudodorsal region of the thorax.

For the cat of the present report, diagnosis of herniation of omental fat was difficult solely with 

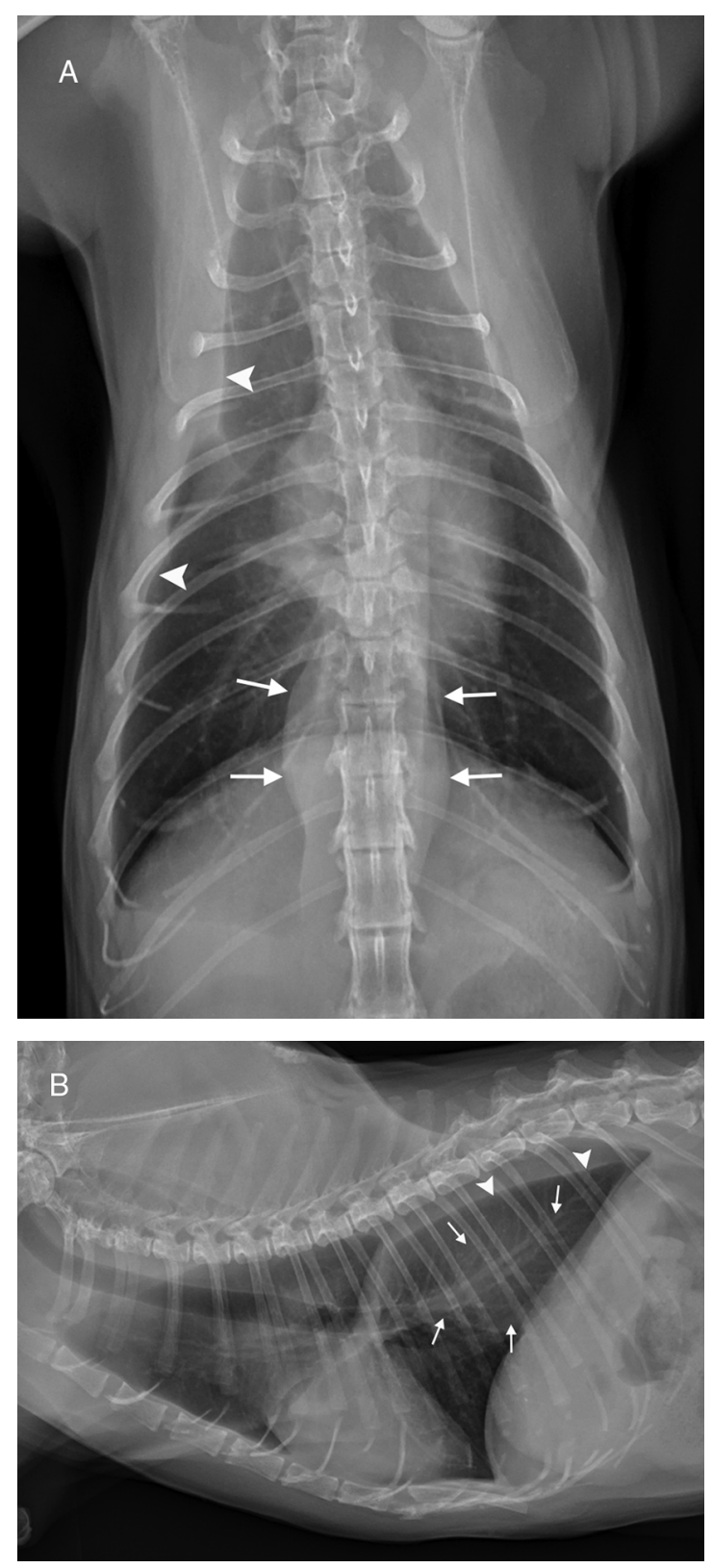

Figure 4-Ventrodorsal (A) and right lateral (B) thoracic radiographic images of the same cat I year later, showing reduced volumes of the hernia (arrows) and pleural fluid or herniated omental fat in the right pleural cavity (arrowheads). radiography. First, the mixed composition of fat and soft tissue of the mass and the caudodorsal location of the mass suggested a fat-containing mass originating from the caudal aspect of the mediastinum. Second, the absence of cranial displacement of abdominal organs was less representative of diaphragmatic hernia. However, we clearly identified the hernia and location of important vessels with the use of pre- and postcontrast CT, which is superior in identifying omental vessels. ${ }^{1,2,4}$ A large volume of inflamed omental fat with prominent blood vessels tightly packed in the caudal mediastinum possibly contributed to the soft tissue opacity seen on radiographic examination of this cat. The exact cause for rupture of the hernia sac was unknown, but a potential trigger for the sac to rupture might have been the catfight 2 days before examination. Once the hernia sac ruptured, the omental fat then extended to the pleural space, and secondary pleural exudate developed. Despite the mid caudodorsal location for the hernia shown on CT, the exact location of the omental hernia could not be ascertained because the owners declined surgical intervention.

\section{Acknowledgments}

The authors received no extrainstitutional funding associated with the present report, and the authors declare that there were no conflicts of interest.

\section{References}

1. Mitsuoka K, Tanaka R, Nagashima Y, Hoshi K, Matsumoto $\mathrm{H}$, Yamane Y. Omental herniation through the esophageal hiatus in a cat. $J$ Vet Med Sci. 2002;64(12):1157-1159. doi:10.1292/jvms.64.1157.

2. Sueyoshi K, Inoue Y, Sumi Y, et al. A case of omental herniation through the esophageal hiatus successfully treated by laparoscopic surgery. Acute Med Surg. 2017;4(3):367-370. doi:10.1002/ams2.288.

3. Tanaka Y, Saika Y, Asao Y, Tanaka M, Nohara R. Omental herniation through the esophageal hiatus: A rare cause of gastric outlet obstruction and its CT findings. Radiol Case Rep. 2020;15(3):269-272. doi:10.1016/j.radcr.2019.10.019.

4. Ruby J, Secrest S, Sharma A. Radiographic differentiation of mediastinal versus pulmonary masses in dogs and cats can be challenging. Vet Radiol Ultrasound. 2020;61(4):385-393. doi:10.1111/vru.12859. 